\title{
Tools for Visual Comparison of Spatial Development Scenarios
}

\author{
Natalia Andrienko, Gennady Andrienko, and Peter Gatalsky \\ Spatial Decision Support Team \\ Fraunhofer Institute AIS \\ Schloss Birlinghoven, 53754 Sankt Augustin, Germany \\ http://ww.ais.fraunhofer.de/SPADE \\ gennady.andrienko@ais.fraunhofer.de
}

\begin{abstract}
In the paper, we suggest a set of visualization-based exploratory tools to support analysis and comparison of different spatial development scenarios, such as results of simulation of various spatially related processes. We have applied a task-analytical approach to tool selection and design, that is, we have first considered what analytical tasks may potentially emerge in the course of investigating and comparing scenarios. We have revealed a set of multifarious tasks, which can be grouped into four categories: 1) analysis in the attribute dimension; 2) analysis in the spatial dimension; 3) analysis in the temporal dimension; 4) spatio-temporal analysis. Then we have evaluated the techniques typically used for visualization of spatial and temporal data, such as (animated) maps or time graphs, from the perspective of supporting different task types. We have extended these techniques, mostly in the direction of increasing interactivity, and combined them for providing a better coverage of the task space. Using examples from the domains of forest management and agriculture, we illustrate how the resulting software tools help in fulfilling various tasks in the course of exploring and comparing scenarios. At the end, we set an explicit correspondence between the task types and the appropriate tools.
\end{abstract}

\section{Introduction}

Simulation is often used for prediction of development of some processes under certain human-controlled conditions. The processes may not only develop in time but also be spread in space. When there is more or less freedom in setting conditions for the development, the question typically arises how to do this in an optimal way. In other words, a decision-maker needs to find among several possible strategies for managing a process such a strategy that maximizes benefit, or minimizes expenses, or decreases ecological damage, etc., or, more often, provides an acceptable trade-off between multiple conflicting goals. For example, in agriculture there is a problem of selection of an appropriate strategy for pesticide application: where, what pesticide, at what time moments, and in what amounts should be applied in order to increase the yield while protecting the soils from dangerous pollution? In forest management, one needs to decide when, where, what tree species, in what age, and in what amount to cut for getting good profit without destroying the forest.

In order to make an informed, well-grounded choice, a decision-maker must have an opportunity to compare results of several simulations. In this paper we consider what visualizations and interactive techniques could be useful for the task of scenario comparison.

Results of spatial process simulation are examples of spatio-temporal data, which may be distinguished on the basis of the character of the changes these data reflect [5]:

- existential changes, i.e. appearance and disappearance of (spatial) objects;

- $\quad$ spatial changes, including changes of position, shape, size, and orientation;

- attribute changes, i.e. changes of non-spatial characteristics of spatial objects.

In this paper, we focus on attribute changes. More specifically, we consider applications where a territory is divided into fixed compartments, and some characteristics of these compartments change over time as a result of human's activities. Thus, in the forest management there are forest compartments with such varying characteristics as the total amount of wood and the structure of the tree mass by species and age categories. In the pesticide application problem, a territory may be divided into parcels according to land use or soil properties, and an analyst is interested in the average pesticide amounts accumulated in the soil and ground waters in each parcel.

From the techniques currently being suggested for visualization of spatio-temporal data, map animation is the most popular [4][8][10][11][13][14]. However, we find that this technique in its "classical" form (i.e. playing a sequence of snapshots representing states of a 
phenomenon at successive moments of time) does not work as well for arbitrary data as it does in demonstrating evident trends like urban growth [15]. The main problem is that animation, in general, is inconvenient for comparing states at different moments in time. In order to detect and evaluate changes, an analyst has to compare the state viewed at the current moment with mental images of earlier states, which need to be kept in mind. Only coherent changes on sufficiently large parts of a territory are well perceived without much cognitive load.

Other techniques suggested for the visualization of spatio-temporal data are map series [14] and change maps [13][14][2]. Spatial and temporal aggregation [9] provides useful summaries of characteristics, but cannot be used alone due to significant information losses involved. In [8] and [12] maps may be complemented by graphs representing changes of individual user-selected spatial objects.

For supporting comparison of development of several spatial phenomena, [8] and [4] suggest simultaneous running of several animations. Animations may be combined in three basic ways [4]: shown in multiple juxtaposed maps, overlaid in the same map, or alternating in the same map. However, to our knowledge, no tests of users' perception of such combined animations have been made yet.

In order to find out which techniques could best support comparison of different spatial development scenarios, let us consider first what basic tasks the process of comparison may consist of.

\section{Possible Tasks in Comparing Scenarios}

Results of a single simulation are values of one or more attributes referring to different territory compartments and time moments, that is, for each compartment there is a temporally ordered series of values of each attribute. In order to compare several simulated scenarios, an analyst needs to derive from this mass of individual values an integral vision of the characteristics of each scenario as a whole. Integration of individual characteristics into an "overall view" [3] occurs in three dimensions: attributive, spatial, and temporal.

In the attributive dimension, when considered separately from the two others, the analyst is interested in properties of the statistical distribution of attribute values: the range of variation, mean or median, dispersion, etc. Often it is important to know the distribution of the values among particular classes, for example, what proportions of the values correspond to low, medium, and high ecological risk.

Integration in the spatial and temporal dimensions is also applied to attribute values but involves also space and time, respectively. In the spatial dimension, characteristics of individual territory compartments need to be integrated into an overall image (pattern) of the spatial distribution of these characteristics. For example, one may observe that attribute values increase from the north to the south, or from the center to the periphery, or that there is a distinct cluster of high values in some part of the territory. When some classes of the characteristics are defined, the analyst is interested whether compartments fitting in the same class are spatially close and form contiguous regions.

In the temporal dimension, the analyst considers sequences of characteristics of each territory compartment as they occur over time. We shall further call such sequences "behaviors". Analyzing a collection of behaviors, one looks whether they are coherent or inconsistent and what patterns/trends prevail. When there are classes of characteristics, an important overall property of a scenario is how the distribution of the values among the classes changes over time. The analyst may be interested in an even higher degree of aggregation, that is, in averaged or summarized values over the whole time period. For example, this may be the total yield in an agricultural scenario or the total costs in an ecological restoration scenario.

In all dimensions, particular attention needs to be paid to so-called outliers. In the attribute dimension, these are occurrences of extremely high or extremely low attribute values standing far apart from the bulk of the values. "Spatial outliers" are compartments considerably differing from the neighborhood by their characteristics. Outliers in the temporal dimension are unusual behaviors, such as abrupt changes of characteristics, or changes that substantially differ from those occurring to the majority of the objects. Typically, outliers not only need to be detected but also explained.

While independent consideration of the spatial and temporal dimensions is a useful part of data analysis, a comprehensive overall view of a scenario requires also their combination. There are two complementary tasks in exploring the evolution of a process in space and time. First, it should be investigated how the pattern of the spatial distribution of the characteristics evolves over time. For example, the analyst might make an observation that the difference between the north and the south increases (decreases) over time, or that a cluster of high values gradually moves in some direction, or spreads, or shrinks. Second, it is important to know how different behavioral patterns are distributed over the territory. For example, it might be found that growing trends prevail in one part of the territory while in the other parts the values tend to decrease or remain relatively stable.

Here is a synopsis of the general characteristics of a scenario an analyst strives to derive: 
1. Time- and space-irrelevant properties characterizing situations at selected time moments: distribution of the individual characteristics in the space of characteristics; various statistical summaries (mean, median, dispersion, variance, etc.); distribution among classes; statistical outliers.

2. Time-irrelevant spatial properties (referring to selected time moments): patterns of spatial distribution of individual characteristics, presence of clusters of compartments with close characteristics, existence of spatial directional trends; spatial distribution of classes; spatial outliers.

3. Space-irrelevant temporal (behavioral) properties: prevailing behavioral pattern or groups of similar behaviors; existence of trends or periodicity; dynamics of value distribution among classes; behavioral outliers; averaged and cumulative values over the period.

4. Spatio-temporal properties:

- Evolution of spatial patterns over time.

- Distribution of behavioral patterns over the territory.

In each particular case, some of these properties may be more relevant than the others, but in any case scenarios are assessed and compared on the basis of general, integrated characteristics rather than attribute values in individual territory compartments at individual time moments. This should be properly taken into account in design or selection of tools for such a kind of analysis. It should be also noted that the initial and final moments of a simulation may play a special role in analyzing simulation results. Usually, the evolution of characteristics is considered in relation to the initial situation. The degree of success or appropriateness of a scenario is often judged from comparing the initial and the final states (although it may be also important that the intermediate states satisfy certain conditions of acceptability). When several scenarios are compared, it is typical that they have the same starting conditions, whereas the further dynamics of the characteristics and, consequently, the final states differ.

Comparison of scenarios requires such tools that could support derivation and comparison of the above-listed integral characteristics. Since it is hardly possible to invent a visualization that would be sufficiently good for all analysis tasks, displays and tools of different types need to be combined. Below we suggest a possible combination of tools. The tools described are implemented in the system CommonGIS (http://www.CommonGIS.de/).

\section{Tools to Analyze and Compare Scenarios}

\subsection{Maps}

Since the processes we deal with develop in space, visualization of their spatial properties is necessary. Maps are suitable media for these purposes. CommonGIS currently offers 16 different techniques for visualization of spatially referenced attribute data on maps. For timedependent data, it is possible to build maps manipulated through time controls, which allow a user to select time moments and view situations at these moments. Hence, for any selected time moment, the user has an opportunity to investigate the corresponding spatial distribution of attribute values. From the cartographic representation methods available in the system, unclassified choropleth map and various classification-based maps are the most suitable for the investigation of spatial patterns: as is recognized in cartography, these techniques promote the perception of a whole territory as a single image, i.e. the required overall view.

The time controls used for the selection of time moments allow the user also to run map animation for observing the evolution of spatial patterns over time. Alternatively, this may be done by visualizing states at different time moments on a series of maps displayed simultaneously. Such maps use the same scheme of graphical encoding of attribute values (e.g. the same color scale). They always have the same size and are zoomed and panned simultaneously. However, when time series are long, the maps may become too small for the patterns to be visible. It may be more reasonable to consider states at selected moments, for example, each fifth or tenth step of the simulation.

The technique of a coherent display of multiple maps (often referred to as "small multiples" [16]) is also good for comparison of several scenarios. The maps can be synchronously manipulated through a common set of time controls. In this way, the user can easily compare states ofifferent scenarios at selected time moments Figure 1).

Another way of representing numeric results of several simulations at selected time moments is showing them on a single map by bar charts, where each bar corresponds to one scenario (see Figure 2). The bars can represent either the original values in the compartments at the corresponding time moments, or differences (ratios) to the initial state, or to the previous time moment. Although such a map cannot support comparison of spatial patterns, it is good for estimating the degree of divergence of the individual characteristics across the scenarios and comparing the amounts of change. By animating the map, one may see whether the characteristics tend to diverge or converge over time. Bar charts can be especially useful in comparing final results of several simulations. 


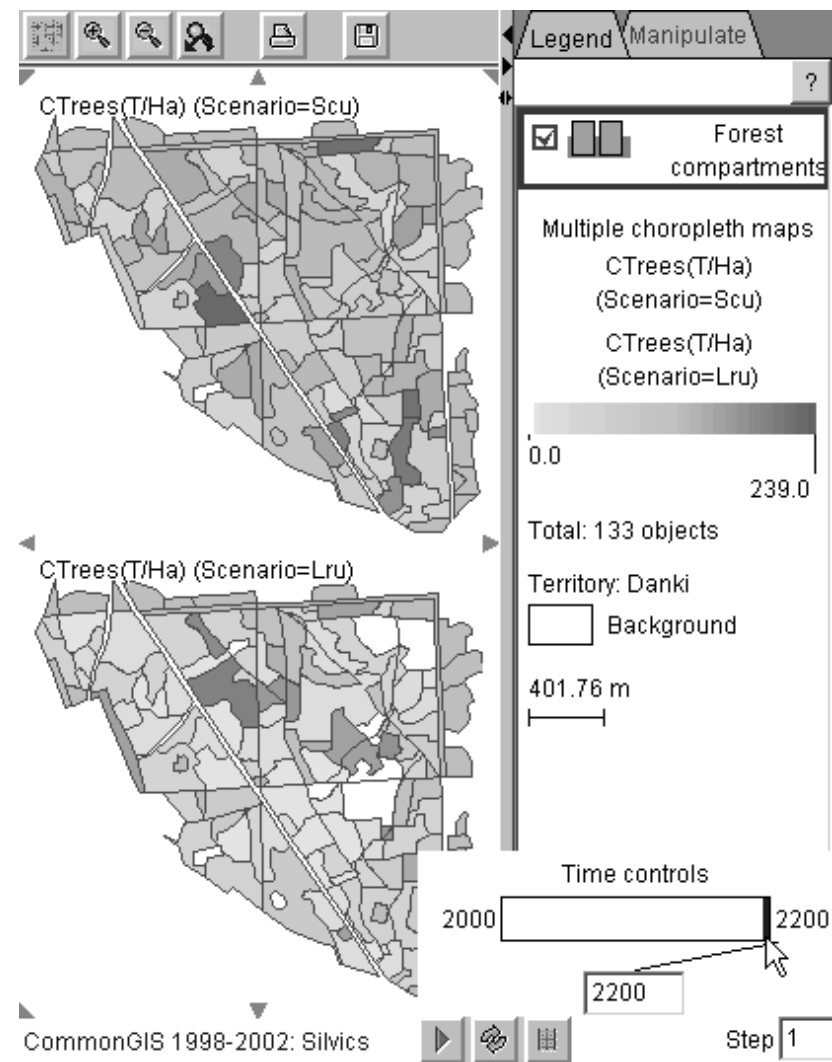

Figure 1. Two choropleth maps, corresponding to different scenarios of forest management, are shown in a common panel and use a common color scale to facilitate comparison. The maps are synchronously manipulated through the time controls, which are superimposed on the lower map, for illustration purposes.

\subsection{Time Graph}

A time graph (Figure 3, top) can be used for visual exploration of numeric data in the temporal dimension. In a time graph, the horizontal axis represents time, and the vertical axis corresponds to values of a numeric attribute. For each spatial object (compartment) the graph contains a line ("value path") built by connecting the positions corresponding to the attribute values attained at consecutive time moments. This line, which can be perceived as a single entity, is a good representation of an individual behavior. For mitigating fluctuations and revealing general patterns and trends, the lines may be smoothed by averaging values over small time intervals.

Since all data displays in CommonGIS are linked through synchronous highlighting of corresponding objects, it is easy to identify which spatial object a particular line belongs to. The work of this dynamic link is illustrated in Figure 3. The possibility to interactively
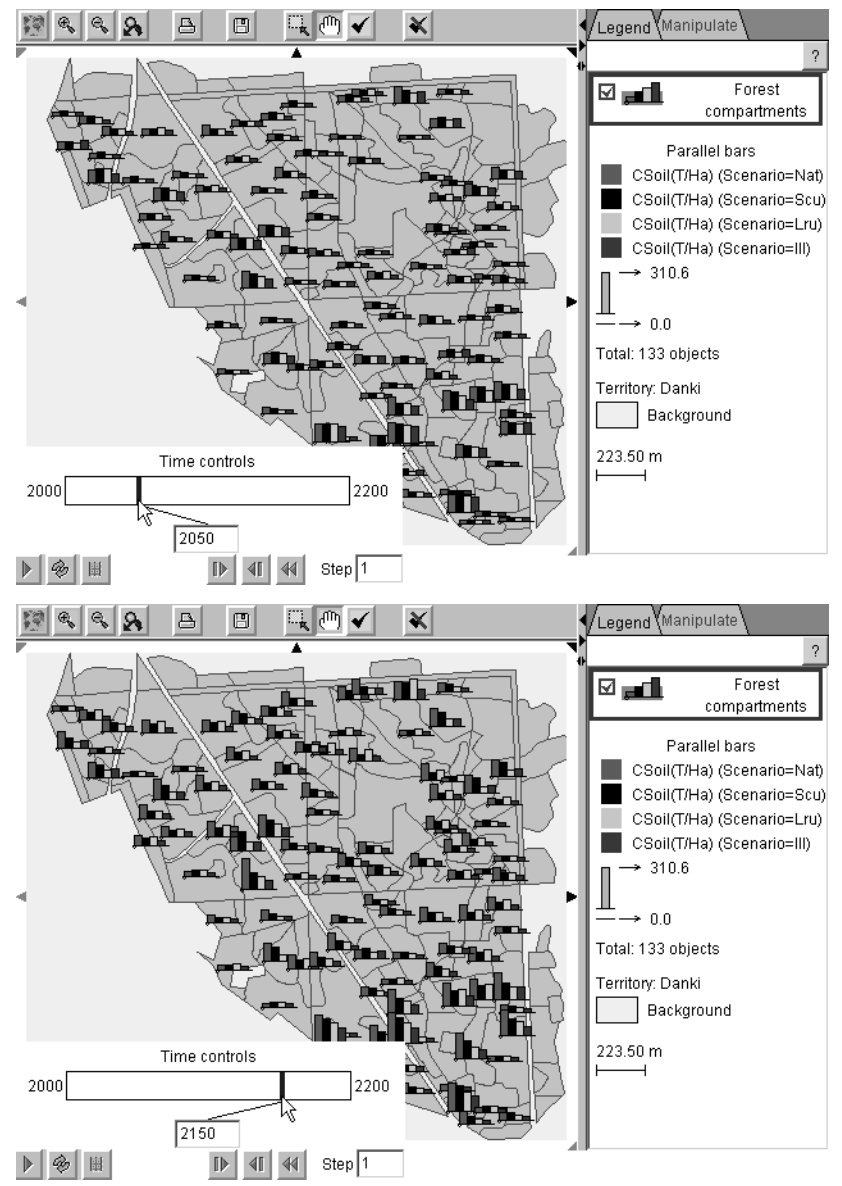

Figure 2. Results of simulation of four different forest management scenarios are represented on a map by bar charts. Shown are states at two time moments, which have been selected using the time controls (superimposed on the maps, for illustration purposes).

select objects in any display allows the user, in particular, to investigate the spatial distribution of behavioral patterns. For this purpose, the user may select groups of neighboring compartments in a map and check on a time graph whether the corresponding behaviors are coherent or diverging. An additional convenience for such an analysis is provided by the possibility to make the time graph show only lines representing selected objects.

Other ways of display linking in CommonGIS are reaction of all displays to changing conditions in a dynamic query and propagation of object colors from a map with some classification of objects to all plots and graphs. Both instruments can be useful in analysis of a set of behaviors.

With a time graph, it is also possible to investigate the statistical distribution of attribute values at each moment and compare distributions at different moments. For this purpose, one needs to look at the crossing positions of the lines representing the behaviors with the vertical grid 
lines (see Figure 3, top). It is easy to see the ranges of variation at each time moment, to estimate the degree of variance, and to detect outliers in the attribute space. However, unusual behaviors may be hard to notice among the multitude of lines, unless they reach extreme values at some moments.



Figure 3. An example time graph represents results of simulation of pesticide concentration in soil compartments. The lines reaching extremely high values on the second step of simulation are selected on the graph using the mouse. As a result, the corresponding soil compartments are highlighted in the map.

Transformations of a time graph help solve this problem and provide additional opportunities for analysis of behaviors. Thus, the user can switch the time graph to the mode of comparison with the previous time moments. In this mode, the graph represents differences or ratios with values for the previous moments rather than the original attribute values (Figure 4). Other possibilities are comparison with values for a fixed, user-selected moment (in particular, with the initial state in a simulation), comparison with a selected object, with average or median values at each time moment, and with a fixed, user-selected value. Transformations of a time graph are discussed in more detail in [2].

In our example shown in Figure 4, a transformed time plot (top) exposes an unusual behavior (the line is highlighted), which is not immediately noticed in the initial variant of the plot (bottom) due to line overlapping. Its unusualness is a sudden drop of the attribute value on step 7 of the simulation followed by a steep rise on step 8 . Hence, graph transformation helped us detect a behavioral outlier.

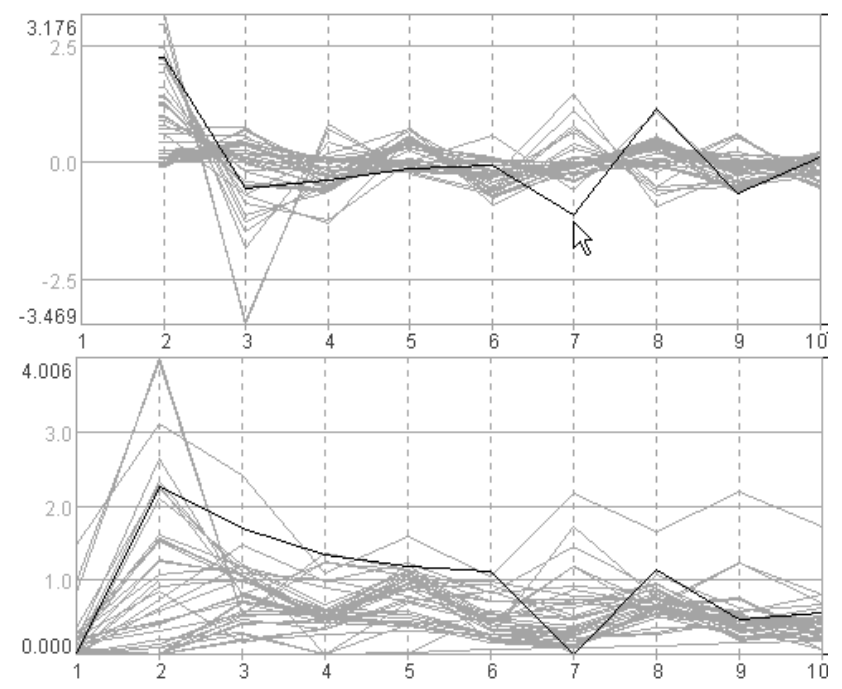

Figure 4. The upper time graph is the result of turning the lower time plot to the mode of comparison with the previous time moments. The highlighted lines in both graphs correspond to the same object.

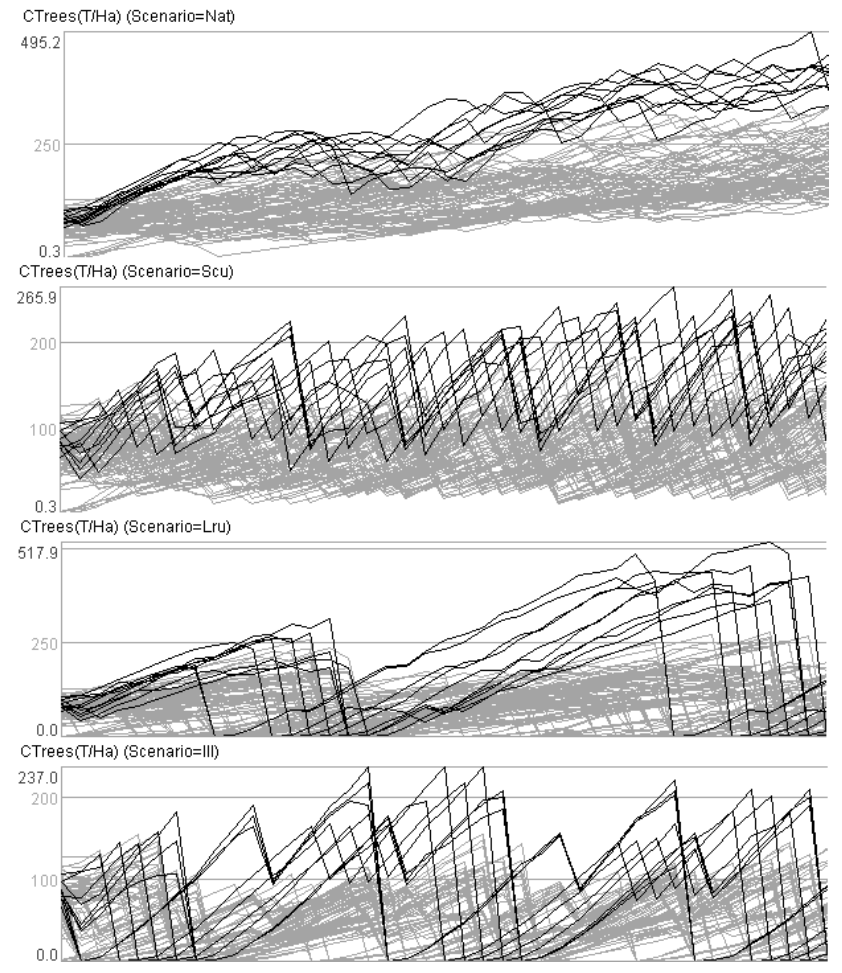

Figure 5. Four juxtaposed time graphs correspond to different forest management scenarios. For a better visibility of the differences in the behaviors, several lines representing the same set of forest compartments are highlighted in all graphs. 
Juxtaposition of several time graphs supports comparing multiple scenarios in the temporal dimension. Thus, Figure 5 demonstrates quite different behavioral patterns of the same attribute in four scenarios of forest management. Besides the patterns, the ranges within which the attribute values vary also differ much.

\subsection{Time Aggregator}

A time aggregator allows the user to divide the value range of a time-dependent numeric attribute into classes (subintervals) and represents graphically the distribution of attribute values among the classes at each time moment. For this purpose, it uses segmented bars (Figure 6). The whole height of a bar corresponds to the total number of objects. The heights of the segments are proportional to the numbers of objects with the values fitting in the respective classes. Such bars are drawn for each time moment. The resulting chart provides an aggregated view of the dynamics of value distribution. The user can easily redefine the classes, for example, for getting a more detailed view with a bigger number of classes.

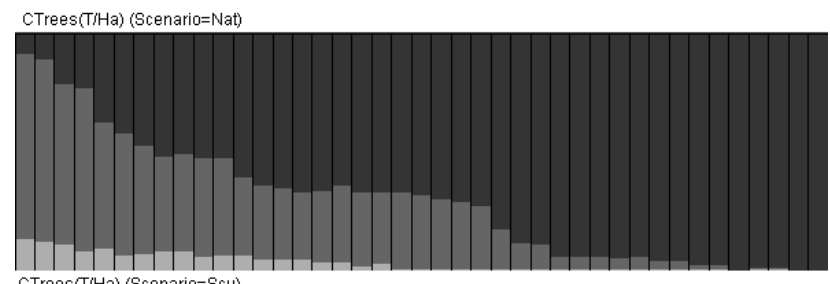

$\mathrm{CTrees}(\mathrm{T} / \mathrm{Ha})(\mathrm{Scenari0}=\mathrm{Scu})$

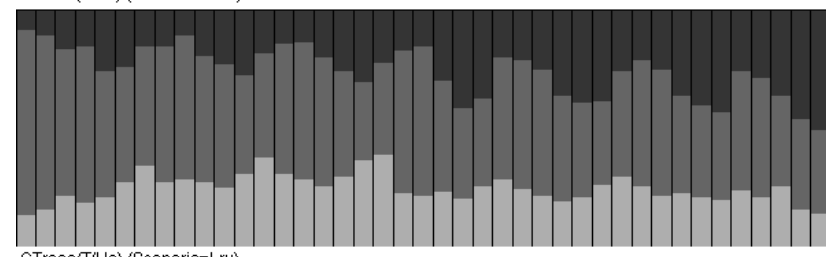

CTrees $(T / H a)$ (Scenario=Lru)

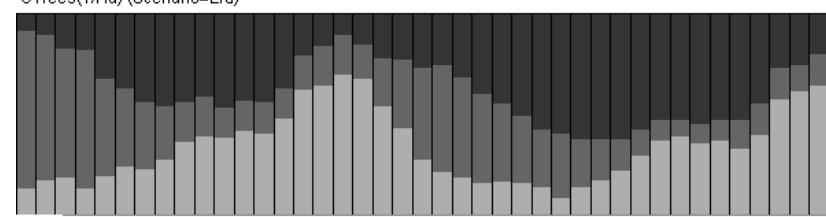

CTrees $(\mathrm{T} / \mathrm{H} \mathrm{H})$ (Scenario=III)

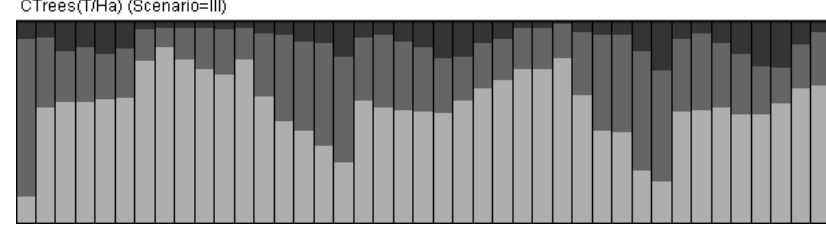

Figure 6. The segmented bars show distribution of attribute values among 3 classes (below 50, 50 to 100, and 100 or more) for each time moment. Charts for four different forest management scenarios are juxtaposed for comparison. The charts represent the same attribute as the time graphs shown in Figure 5.
Class aggregation charts are convenient for comparing scenarios. For example, in Figure 6, the same division into classes (below 50, 50 to 100 , and 100 or more) has been applied to the same attribute in four forest management scenarios. The lower, light bar segments correspond to values below 50, the segments in the middle to the class 50 to 100 , and the upper, dark segments to the class 100 and more. The differences between the scenarios are very well visible. While a detailed consideration of the meaning of these differences is beyond the scope of the paper, we would like to give here a brief clarification. The attribute approximately represents the available amount of wood. The first scenario corresponds to a natural forest growth, i.e. without forest cuts, the three others simulate different strategies of cutting. From Figure 6 it may be seen that the first strategy (i.e. the second scenario) is the closest to the idea of a sustainable forest management while the third strategy significantly reduces the wood reserve (at some moments there are almost no compartments with values 100 and more) and can eventually completely destroy the forest.

\subsection{Other Tools}

Additionally to the visual tools described above, CommonGIS provides a variety of other instruments that may be also useful in analysis of spatio-temporal data and, in particular, in comparing scenarios. Thus, various types of graphical displays (dot plots, histograms, scatter plots, parallel coordinate plots) may be helpful in comparing results of several simulations at selected time moments, for example, the final results. Investigations in the attribute dimension can be also supported by computational tools, which derive various statistics such as mean, median, and other percentiles, including statistics for any user-defined classes of objects. An analyst can also apply the dynamic query tool for focusing the investigation process on data subsets with particular characteristics. For example, the analyst may wish to see what will happen to forest compartments having high initial reserve of wood in different scenarios of forest management. For this purpose, the analyst needs to set appropriate conditions in the dynamic query. In response, all displays currently present on the screen will hide objects that do not satisfy these conditions. In particular, the time graphs corresponding to the different scenarios will represent attribute dynamics only in the forest compartments satisfying the query thus making the comparison more convenient.

\section{Task-Tool Correspondence}

In Section 2 we gave an overview of analytical tasks that may potentially arise in analysis and comparison of 
spatial development scenarios. The tasks were grouped into four categories: 1) analysis in the attribute dimension, i.e. investigation of space- and time-irrelevant properties; 2) analysis in the spatial dimension, i.e. investigation of spatial distribution of attribute values irrespective of time; 3) analysis in the temporal dimension, i.e. analysis of behaviors with no regard to space; 4) spatio-temporal analysis, i.e. development of spatial patterns over time and spatial distribution of behaviors. In Section 3 we introduced a number of tools suggested to support these kinds of analysis. Here we would like to state explicitly which tools are appropriate for which tasks.

Analysis in the attribute dimension. For any particular time moment, distribution of individual characteristics in the space of characteristics can be investigated using various graphical displays. For a single numeric attribute, an analyst can apply the dot plot, stacked dot plot, or histogram. The scatterplot display is appropriate for two numeric attributes while the scatterplot matrix and the parallel coordinate plot could be used for multiple attributes. The histogram display provides the most aggregated view of a distribution. Due to the dynamic linking of the displays in CommonGIS, several histograms may be used for simultaneous analysis of multiple attributes. Analysis in the attribute dimension is also supported by statistical computations, which include, among others, techniques suitable for qualitative data. CommonGIS supports various methods of classification, can propagate resulting classes to all displays (the objects in the displays are colored according to the classes they belong to), and computes statistics for the classes.

Spatial analysis. The primary tools for exploring and comparing patterns of spatial distribution of attribute values are maps. However, patterns can be well perceived when data are represented by means of area coloring rather than by using charts or other symbols, but almost all coloring-based visualization techniques are only suitable for a single attribute, except for the crossclassification applied to two numeric attributes [6][1]. When it is necessary to investigate the distribution of complex characteristics expressed by multiple attributes, it can be recommended to use the opportunities provided by the dynamic link between displays. Thus, the user can select regions on a map and see where the corresponding objects are on a matrix of scatterplots or on a parallel coordinate plot. The user can also select dots or lines in a plot or bars in a histogram and see how the corresponding objects are located spatially. The dynamic query tool also allows the user to locate particular characteristics in space. Comparison of different scenarios at selected time moments is best supported by the coordinated display of multiple maps in a common panel.
Temporal analysis. Analysis in the temporal dimension can be done using time graphs and time aggregation charts, as was described in $\S \S 3.2$ and 3.3 . The time aggregation chart provides a more general view of a scenario but conceals important information about existing behavioral patterns. For better understanding of the patterns it is recommended to apply transformations of the time graph, i.e. the different modes of visual comparison. Averaged and cumulative values over time period can be obtained using the computational facilities of CommonGIS.

Spatio-temporal analysis. Evolution of spatial patterns over time may be investigated by applying usercontrolled map animation. It is also possible to visualize states at several moments on a series of maps. Comparison of pattern development in different scenarios is supported by synchronous animation of several maps. An animated barchart map with bars corresponding to the scenarios allows the user to estimate local differences and their variation over time.

The complementary task of the spatio-temporal analysis, investigation of spatial distribution of behavioral patterns, is supported by the dynamic link between maps and time graphs. The user may select regions on a map and see the corresponding patterns on a time graphs or on several time graphs corresponding to different scenarios. The user may also select lines on a graph representing similar behaviors and see on a map whether the corresponding compartments are spatially close.

\section{Conclusion}

We have applied a task-analytical approach in order to create an appropriate collection of tools for supporting visual investigation and comparison of spatial development scenarios, such as results of simulation of spatially related processes. There is an experience of applying this tool collection to analysis of forest management scenarios. The domain experts who did this comparative study expressed their high appreciation of the tools. Some results of this analysis are described in [7]. The experts continue using the tools in combination with their simulation models within the EU-funded INTAS project Silvics. It is also planned to apply the exploratory tools of CommonGIS in another EU-funded project, GIMMI (IST-2001-34245, 2002-2004), for analysis of simulation results concerning application of various pesticides in agriculture and the resulting environmental impact.

We realize that it is necessary to conduct usability studies in order to check whether the tools are understandable and convenient for users and whether they effectively support the tasks they are designed for. We intend to do such studies in the future and would be 
happy to cooperate in these activities with interested researchers and organizations.

\section{Acknowledgements}

This work was partly supported within the EU-funded project GIMMI. The simulation results used in this paper for illustration purposes were provided by our partners in the projects Silvics (http://www.efi.fi/projects/silvics/) and GIMMI (http://services.txt.it/gimmi/).

\section{References}

[1] G. Andrienko and N. Andrienko, Interactive maps for visual data exploration. International Journal Geographical Information Science 13, 1999, pp.355-374

[2] N. Andrienko, G. Andrienko, and P. Gatalsky, "Exploring Changes in Census Time Series with Interactive Dynamic Maps and Graphics". Computational statistics, 16 (3), 2001, pp.417433.

[3] J. Bertin, Semiology of Graphics. Diagrams, Networks, Maps, The University of Wisconsin Press, Madison, 1983.

[4] C. Blok, B. Koebben, T. Cheng, and A.A. Kuterema, "Visualization of Relationships Between Spatial Patterns in Time by Cartographic Animation". Cartography and Geographic Information Science 26 (2), 1999, pp 139-151

[5] C. Blok, "Monitoring Change: Characteristics of Dynamic Geo-spatial Phenomena for Visual Exploration". In Freksa Ch et al. (eds) Spatial Cognition II, LNAI 1849, Springer-Verlag, Berlin/Heidelberg, 2000, pp 16-30

[6] C.A. Brewer, "Color use guidelines for mapping and visualization", In Visualisation in Modern Cartography (New York: Elsevier Science Inc.), 1994, 123-147.

[7] O. Chertov, A. Komarov, G. Andrienko, N. Andrienko, and P. Gatalsky, "Integrating forest simulation models and spatial-temporal interactive visualization for decision making at landscape level", Ecological Modelling, v.148 (1), 2002, pp.4765

[8] R. Edsall and D. Peuquet, "A Graphical User Interface for the Integration of Time into GIS". Proceedings of the 1997 American Congress of Surveying and Mapping Annual Convention and Exhibition, Seattle, WA, 1997, pp.182-189.

[9] A. Fredrikson, C. North, C. Plaisant, and B. Shneiderman "Temporal, geographic and categorical aggregations viewed through coordinated displays: a case study with highway incident data". In Proceedings of Workshop on New Paradigms in Information Visualization and Manipulation (NPIVM'99), New York: ACM Press, 1999, pp 26-34

[10] M. Harrower, A.M. MacEachren, and A.L. Griffin, "Developing a Geographic Visualization Tool to Support Earth Science Learning". Cartography and Geographic Information Science 27 (4), 2000, pp 279-2

[11] M.-J. Kraak, R. Edsall, and A.M. MacEachren, "Cartographic animation and legends for temporal maps: exploration and/or interaction". In Proceedings 18th International Cartographic Conference, Vol 1, 1997, pp 253261

[12] K. Morris, D. Hill, and T. Moore, "Integrated data management and visualization through space and time". In $K$. Fullerton (ed.): Proceedings of $5^{\text {th }}$ EC-GIS Workshop, Stresa, Italy, 28-30 June 1999, EUR 19018 EN, European Communities, pp. 460-473

[13] C. Oberholzer and L. Hurni, "Visualization of change in the Interactive Multimedia Atlas of Switzerland", Computers \& Geosciences, 26 (1), 2000, pp 423-435

[14] T. Slocum, S. Yoder, F. Kessler, and R. Sluter "MapTime: Software for Exploring Spatiotemporal Data Associated with Point Locations", Cartographica 37 (1), 2000, pp 15-31

[15] W. Tobler, "Computer movie simulating urban growth in the Detroit region”. Economic Geography, 46 (2), 1970, pp 234240

[16] E.R Tufte, The Visual Display of Quantitative Information, Cheshire: Graphics Press, 1983. 